\title{
Time Reversal Synthetic Aperture Radar Imaging In Multipath
}

\author{
Yuanwei Jin, José M.F. Moura, and Nicholas O’Donoughue \\ Electrical and Computer Engineering \\ Carnegie Mellon University \\ Pittsburgh, PA $15213^{*}$ \\ Michael T. Mulford and Alphonso A. Samuel \\ Raytheon Missile Systems \\ Tucson, AZ 85734
}

\begin{abstract}
Conventional spotlight Synthetic Aperture Radar (SAR) assumes a single reflection of transmitted waveforms from targets [1]. Multiple reflections of targets due to surrounding scatterers appear as ghosting artifacts in conventional SAR images, which obscures true target image and leads to poor resolution. In this paper, we develop image formation techniques using time reversal, Time Reversal SAR (TR$S A R)$, to remove ghosting artifacts and achieve high resolution. The TR-SAR algorithm is tested using phase history data collected by a rail-mounted SAR sensor operated by Raytheon.
\end{abstract}

\section{Introduction}

Many data adaptive high resolution approaches to synthetic-aperture radar (SAR) image reconstruction have been developed [2,3]. Our work is concerned with the exploitation of time reversal in spotlight synthetic aperture radar imaging in multipath rich environments. For targets in a rich scattering environment, conventional SAR results in ghosting artifacts due to multiple bounces by surrounding scatterers. To counter this, we develop time reversal SAR algorithms to process phase history data in rich multipath scattering. The ultimate goal of TR-SAR is to improve automated target recognition and to aid image analysts in identifying targets in dense multipath.

We developed the TR-SAR algorithm in [4] for target focusing and ghost image removal. We show in [4], how, from a rough estimate of the target location obtained from a conventional SAR image and using time reversal, TR-SAR focuses on the target with improved resolution, and reduces or removes ghost images. TR-SAR uses two-pass collections to perform a sensitive form of change detection - detecting targets by using two SAR images that capture the same

\footnotetext{
*This work is funded by the Defence Advanced Research Projects Agency through the Army Research Office under grant no. W911NF-04$1-0031$
}

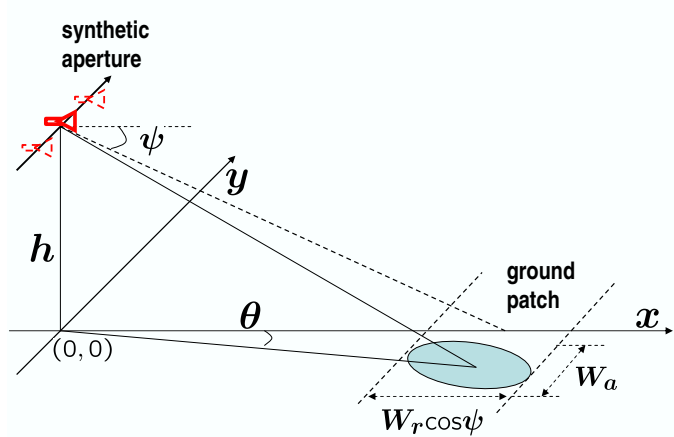

Figure 1. Spotlight SAR imaging geometry.

scene. In this paper, we focus on TR-SAR algorithms for linear frequency modulated (LFM) chirp signals. For a SAR radar that uses LFM chirp signals, radar returns undergo stretch processing and IF sampling to obtain the phase history data. Stretch processing, a commonly used technique for pulse compression in radar, enables narrowband sampling for wideband waveforms and converts range (i.e., fast time) into the spatial frequency domain $[5,6]$. We verify the proposed imaging algorithms successfully using electromagnetic experimental data collected by a rail-mounted SAR sensor at Raytheon in a rich scattering environment.

\section{Mathematical Description of TR-SAR}

For illustration purposes, we use the mono-static configuration shown in Fig. 1, where a SAR sensor images the ground patch at range $x$ and cross range $y$. For the SAR imaging platform at height $h, \psi$ and $\theta$ are the depression angle and the squint angle, respectively; The symbols $W_{r}$ and $W_{a}$ denote the range swath and the azimuth swath, respectively. Next, we describe the time reversal signal model in stretch processing. 


\subsection{Time Reversal in Stretch Processing}

Linear frequency modulated chirp signals take the form of

$$
s(t)=\cos \left(2 \pi f_{c} t+\pi \gamma t^{2}\right), \quad|t| \leq T_{p} / 2
$$

where $\gamma$ is the chirp rate in units of radian $/ \sec ^{2}, T_{p}$ is the waveform duration, and $f_{c}$ is the carrier frequency. The radar transmits a sequence of such LFM signals with an interpulse period of $T$. The pulse repetition frequency PRF is $1 / T$. These pulses have the form of $\mathfrak{R}\{s(n, t)\}$, where the complex exponential form of the transmitted signal can be written as follows [1]:

$$
s(n, t)=\operatorname{rect}\left(\frac{t-n T}{T_{p}}\right) e^{j\left[2 \pi f_{c} t+\pi \gamma(t-n T)^{2}\right]} .
$$

The quantity $n$ is the pulse number; $n=0$ represents the first transmitted pulse. Let $\widetilde{t}=t-n T$ denote the time within a signal received from a specific transmitted pulse and referenced to the time of pulse generation. The function $\operatorname{rect}(u)=1$ for $|u| \leq \frac{1}{2}$ is the rectangular window function. The illuminating signal $s(t)$ is emitted from the transmit antenna, propagates to the target scene, and is reflected back to the receive antenna. The received signal from a single point target can be written as

$$
s_{r}(n, \widetilde{t})=A_{t} \operatorname{rect}\left(\frac{\tilde{t}}{T_{p}}\right) e^{j\left[2 \pi f_{c}(t-\tau)+\pi \gamma(\widetilde{t}-\tau)^{2}\right]},
$$

where $A_{t}$ is the target reflectivity and $\tau$ is the round-trip propagation delay. Next, the SAR we use employs stretch processing for pulse compression. Stretch processing is commonly used in high resolution radar and target recognition [5, 6]. Stretch processing has two basic features: (1) it enables the use of wideband waveform with narrowband processing. For example, for the rail-SAR system we use, the sampling rate is $300 \mathrm{MHz}$ while the chirp signals have 1 $\mathrm{GHz}$ bandwidth; (2) it converts range into spatial frequency. This is because the received signal is mixed with a reference signal that has the same chirp rate as the transmitted waveform. Given the reference range $R_{0}$, i.e., the distance between the antenna phase center to the target scene center, the reference signal is

$$
s_{\mathrm{ref}}(n, \widetilde{t})=e^{j\left[2 \pi f_{c}\left(t-\frac{2 R_{0}}{c}\right)+\pi \gamma\left(\widetilde{t}-\frac{2 R_{0}}{c}\right)^{2}\right]} .
$$

The target returns are translated into tones by taking the difference frequency out of the mixer. The frequency of the tones denotes the ranges to the targets. The mixing operation results in a pulse compressed signal

$$
\begin{aligned}
s_{c}(n, \widetilde{t})= & s_{r}(n, \widetilde{t}) s_{\text {ref }}^{*}(n, t) \\
= & A_{t} \operatorname{rect}\left(\frac{\tilde{t}}{T_{p}}\right) \underbrace{e^{-j 2 \pi\left[f_{c}+\gamma\left(\widetilde{t}-\frac{2 R_{0}}{c}\right)\right] \frac{2\left(R_{t}-R_{0}\right)}{c}}}_{\text {phase term }} \\
& \underbrace{e^{j 4 \pi \gamma \frac{\left(R_{t}-R_{0}\right)^{2}}{c^{2}}}}_{\text {RVP }} .
\end{aligned}
$$

The residual video phase (RVP) is an unwanted artifact arising from the range dechirping process and can be removed [1]. Ideally, the reference range $R_{0}=R_{0}(n T)$ is the exact range from the antenna phase center to the selected scene center at each pulse transmission time. In real SAR imaging systems, $R_{0}$ is not precisely equal to the round trip propagation time to the center of the ground patch. Thus, post-processing techniques, such as autofocus, or automatic phase-error correction, are employed [7]. For simplicity, in what follows, we ignore the quadratic phase term. Thus, we have

$$
s_{c}(n, \widetilde{t})=A_{t} \operatorname{rect}\left(\frac{\tilde{t}}{T_{p}}\right) e^{-j 2 \pi\left[f_{c}+\gamma\left(\widetilde{t}-\frac{2 R_{0}}{c}\right)\right] \frac{2\left(R_{t}-R_{0}\right)}{c}} .
$$

where the common time window is given by

$$
-\frac{T_{p}}{2}+\frac{W_{r}}{c} \leq t-n T-\frac{2 R_{0}}{c} \leq \frac{T_{p}}{2}-\frac{W_{r}}{c} .
$$

Furthermore, the phase term in (7) can be decomposed as a product of the instantaneous spatial frequency

$$
U_{n}=4 \frac{\pi\left(f_{c}+\gamma\left(\widetilde{t}-\frac{2 R_{0}}{c}\right)\right)}{c}
$$

and the range distance $\left(R_{t}-R_{0}\right)$. By (8), the spatial frequency (9) can be written as

$\frac{4 \pi}{c}\left(f_{c}+\gamma\left(-\frac{T_{p}}{2}+\frac{W_{r}}{c}\right)\right) \leq U_{n} \leq \frac{4 \pi}{c}\left(f_{c}+\gamma\left(\frac{T_{p}}{2}-\frac{W_{r}}{c}\right)\right)$

with the spatial frequency bandwidth $\left(\mathrm{m}^{-1}\right)$ given by

$$
B_{U}=\frac{\gamma}{c}\left(T_{p}-\frac{2 W_{r}}{c}\right)=2\left(B_{c}-B_{\mathrm{IF}}\right) / c \approx 2 B_{c} / c .
$$

The approximation is due to $B_{c} \gg B_{\mathrm{IF}}$ for practical SAR systems using stretch processing. Closely examining (7) reveals that

$$
\begin{aligned}
& e^{-j 2 \pi\left(f_{c}+\gamma\left(\widetilde{t}-\frac{2 R_{0}}{c}\right)\right) \frac{2\left(R_{t}-R_{0}\right)}{c}}=e^{-j U_{n}\left(R_{t}-R_{0}\right)} \\
= & \int \delta\left(u(n, \widetilde{t})-\left(R_{t}-R_{0}\right)\right) e^{-j u U_{n}} d u \\
= & \mathcal{F}_{u}\left\{\delta\left(u(n, \widetilde{t})-\left(R_{t}-R_{0}\right)\right)\right\} .
\end{aligned}
$$

It is well known that time reversal in the time domain is equivalent to phase conjugation in the frequency domain. In our case, time reversal of the demodulated signal can be treated as phase conjugation in the spatial frequency domain. Use of linear FM chirp and stretch processing translates the pulse compressed signal into Fourier transform in range (spatial) domain. Thus, range is converted into spatial frequency. As a result, phase conjugation can be applied directly to the pulse compressed signal. The time-reversed dechirped signal (after removing RVP) can be written as

$$
\left[s_{c}(n, \widetilde{t})\right]^{*}=A_{t}^{*} \operatorname{rect}\left(\frac{\tilde{t}}{T_{p}}\right) e^{j U_{n}\left(R_{t}-R_{0}\right)}
$$


with the target response filter $A_{t} e^{-j U_{n}\left(R_{t}-R_{0}\right)}$ confined within the window specified by $\operatorname{rect}\left(\frac{\tilde{t}}{T_{p}}\right)$. This observation enables a simple implementation of time reversal in SAR with stretch processing. The chirp signal will not be involved in the subsequent mathematical time-reversal transmission. Next, we discuss the TR-SAR processing.

\subsection{TR-SAR Description}

The proposed spotlight mode TR-SAR consists of four major steps: (1) Clutter probing; (2) Target probing; (3) Time reversal; (4) Multi-look averaging and TR-SAR image formation.

Step-1: Clutter probing This is the first step in a two-pass data collection process. The target is not present. A LFM chirp signal is sent to probe the clutter environment. We then record the phase history data as the reference data.

Step-2: Target probing This is the second step in the twopass data collection process. The target is now present. A LFM chirp signal is sent to probe the target environment, which results in the test data. The signal returns recorded in the first step will be subtracted out from this step. The subtraction procedure will remove the strong reflections from dominant scatterers in the vicinity of the target. In a multipath rich environment, the compressed return signal is the superposition of the direct path from the target and the multiple reflection due to the surrounding scatterers, which takes the form of

$$
s_{c}(n, \tilde{t})=\operatorname{rect}\left(\frac{\tilde{t}}{T_{p}}\right) \sum_{l=0}^{L-1} A_{l} e^{-j U_{n}\left(R_{t_{l}}-R_{0}\right)},
$$

where $A_{l}$ is the reflectivity for each reflection; $R_{t_{l}}$ is the nominal slant range for each reflection given by

$$
\begin{aligned}
R_{t_{l}}(n, t)= & {\left[\left(x_{l}-x_{a}(n, t)\right)^{2}+\left(y_{l}-y_{a}(n, t)\right)^{2}\right.} \\
& \left.+\left(z_{l}-z_{a}(n, t)\right)^{2}\right]^{-1 / 2}
\end{aligned}
$$

where $\left(x_{0}, y_{0}, z_{0}\right)$ is the (direct path) target location; $\left(x_{l}, y_{l}, z_{l}\right), l=1, \cdots, L-1$ are the coordinates of each multipath reflection. $x_{a}(n, t), y_{a}(n, t)$, and $z_{a}(n, t)$ denotes the antenna position at pulse $n$ and time $t$ in range, cross range, and elevation, respectively.

Next, we construct the target impulse response function (IPR) based on the initial target range estimation

$$
s_{0}(n, \widetilde{t})=\operatorname{rect}\left(\frac{\tilde{t}}{T_{p}}\right) \widehat{A}_{0} e^{-j U_{n}\left(\widehat{R}_{t_{0}}-R_{0}\right)}
$$

where $\widehat{A}_{0}$ and $\widehat{R}_{t_{0}}$ is the reflectivity and the distance of the estimated target to be focused on. The estimation requires the multilook processing discussed in section 2.3. In practice, the antenna moves along the track continuously. For simplicity, we make assume that the antenna is stationary during pulse transmission and reception while moving in discrete increments between pulses. For SAR, the measurement data are collected via a platform that carries the radar. Suppose that the speed of this platform is $v$, the slow-time interval between successive radar transmissions is the pulse repetition interval (PRI) (pulse repetition frequency or PRF is $1 / T) T=\frac{\Delta_{y}}{v}$, where $\Delta_{y}$ is the distance that the radar travels during each transmission. In a practical radar system, the PRF is usually small. For example, the rail-SAR system at Raytheon has PRF $=8 \mathrm{~Hz}$, and $v=6 \mathrm{~cm} / \mathrm{sec}$. Hence, $\Delta_{y}=0.75 \mathrm{~cm}$. This implies that the position of the antenna at each pulse can be written as

$$
x_{a}(n, t)=x_{a}(n)=\frac{v}{\operatorname{PRF}}\left(n+n_{o}\right), n=0, \cdots, N-1
$$

where $n_{o}$ is an offset and needs to be determined by calibration. We further assume that all targets are in the ground plane with $z_{t}=0$. Thus, the range term takes the form of

$$
\widehat{R}_{t_{0}}(n, t)=\sqrt{\left(\widehat{x}_{t}-\left(n+n_{o}\right) v T\right)^{2}+\widehat{y}_{t}^{2}+\left(R_{c} \sin \psi\right)^{2}}
$$

Step-3: time reversal In the time reversal step, the return signal is time-reversed, phase conjugated, and energy normalized. Let $\mu(n)$ be the energy normalization factor for each pulse, thus, the time-reversed signal becomes

$$
s^{\operatorname{tr}}(n, \widetilde{t})=\mu(n) \operatorname{rect}\left(\frac{\tilde{t}}{T_{p}}\right) \sum_{l=0}^{L-1} A_{l}^{*} e^{j U_{n}\left(R_{t_{l}}-R_{0}\right)} .
$$

The returned signal of this re-transmitted signal becomes

$$
s_{r}^{\operatorname{tr}}(n, \tilde{t})=\operatorname{rect}\left(\frac{\tilde{t}}{T_{p}}\right)\left|\mu(n) \sum_{l=0}^{L-1} A_{l}^{*} e^{j U_{n}\left(R_{t_{l}}-R_{0}\right)}\right|^{2} .
$$

Step-4: TR-SAR processing The TR-SAR processing is confined to a selected window illustrated in Fig. 3. The image is processed in small image chips. We use windowing to remove the interference caused by very strong reflectors, for instance, trihedral corner reflectors, and to localize the multipath reflection in the region of interest (ROI). The selected image chip undergoes the windowed inverse Fourier transform to extract the signal for subsequent time reversal processing. By matching the returned time-reversed signal by the estimated target impulse response filter, we obtain the following data for image reconstruction:

$$
x^{\mathrm{re}}(n, \widetilde{t})=s_{r}^{\mathrm{tr}}(n, \widetilde{t}) s_{0}(n, \widetilde{t})
$$

\subsection{Multi-look Averaging}

Multilook averaging is a necessary step in the proposed TR-SAR algorithm to remove random ghost patterns in SAR images with multipath [8]. In multi-look (subaperture) processing, different subapertures of a ground scene 


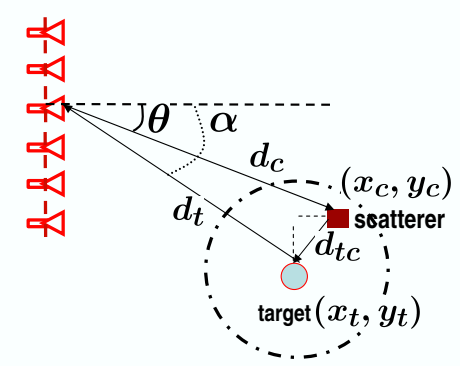

Figure 2. Ghost artifacts in rich scattering

form different subaperture images [7]. In a rich scattering environment where there are many scattering objects in the vicinity of the target, the ghost patterns caused by the multipath will be look angle dependent. The independence between various subaperture images can reduce or remove ghost pattern artifacts caused by multipath reflections.

\subsubsection{Analysis of Ghost Patterns}

Fig. 2 shows the effect of multipath due to random placement of a scatterer near a target. The single reflection returned signal travels a total distance of $d_{c}+d_{t c}+d_{t}$. This returned signal induces ghost artifacts. To characterize the location of the ghost artifacts that appear on a SAR image, we calculate the ghost range $x_{g}$ and the cross range $y_{g}$. We define the target coordinates

$$
x_{t}=d_{t} \cos \alpha, y_{t}=d_{t} \sin \alpha
$$

where $\alpha$ is the aspect angle of the target with respect to the synthetic aperture. Hence, the scatterer coordinates are

$$
x_{c}=d_{c} \cos \theta, \quad y_{c}=d_{c} \sin \theta
$$

where $\theta$ is the aspect angle of the scatterer with respect to the antenna. By Taylor series expansion, we have

$$
\left\{\begin{array}{l}
\cos (\alpha+\Delta \theta)=\cos \alpha-\sin \alpha \Delta \theta+O\left(\Delta^{2} \theta\right) \\
\sin (\alpha+\Delta \theta)=\sin \alpha+\cos \alpha \Delta \theta+O\left(\Delta^{2} \theta\right)
\end{array}\right.
$$

Let $d_{g}=\left(d_{t}+d_{c}+d_{t c}\right) / 2$ and define

$$
x_{g}=d_{g} \cos \alpha, y_{g}=d_{g} \sin \alpha
$$

we have

$$
\left\{\begin{array}{l}
x_{g}=\frac{x_{t}+x_{c}}{2}+\frac{y_{c}}{2} \Delta \theta+\frac{d_{t c}}{2} \cos \alpha \\
y_{g}=\frac{y_{t}+y_{c}}{2}-\frac{x_{c}}{2} \Delta \theta+\frac{d_{t c}}{2} \sin \alpha
\end{array}\right.
$$

In a SAR scenario, the cross range $y_{c} \ll x_{c}$, and $\alpha$ is a small angle, i.e., $\cos \alpha \approx 1, \sin \alpha \approx \alpha$. Therefore, we have the following approximation

$$
\left\{\begin{array}{l}
x_{g} \approx \frac{x_{t}+x_{c}}{2}+\frac{d_{t c}}{2} \\
y_{g} \approx \frac{y_{t}+y_{c}}{2}-\underbrace{\frac{x_{c}}{2} \Delta \theta}_{\text {scattering spread }}+\underbrace{\frac{d_{t c}}{2} \alpha}_{\text {scattering density }}
\end{array}\right.
$$

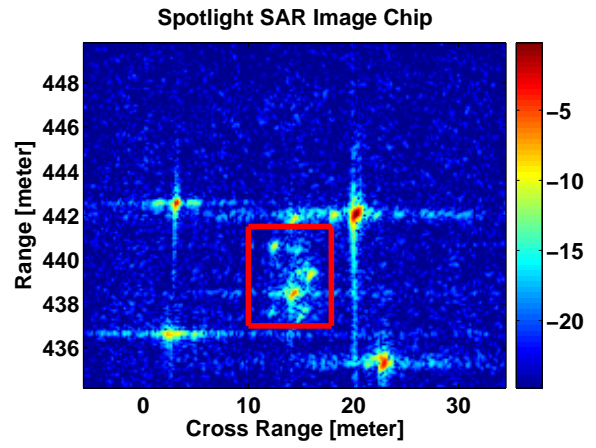

Figure 3. SAR image Chip

Eqn. (26) shows that the ghost artifacts in cross range $y_{g}$ depend on the scattering spread $(\Delta \theta)$ and the scattering density $\left(\alpha\right.$ and $\left.d_{t c}\right)$. The analysis demonstrates that the appearance of the ghost pattern artifacts is look angle $\alpha$ dependent. Since the true target location is fixed, averaging multi-look images, either coherently or non-coherently, will reduce or remove the ghost patterns and enhances the intensity of the target. Hence, from the averaged multilook images, we obtain a rough estimate of the target location with a coarse resolution. Later, the estimated target location can be used for reconstructing the TR-SAR images in the full aperture domain to retain full resolution.

\section{Field Experiments at the Raytheon Site}

We test the proposed algorithms using the phase history data collected by a rail-mounted radar system in a desert area at Raytheon, Tucson, Arizona. The data collection was conducted in August - October 2007. The target area is an open field at 440 meters down range of the radar. In the open field, we constructed a square-shaped test site of 20 meters wide and 7 meters deep. Four trihedral corner reflectors are placed at the four corners of the test site for registration purposes. Within this test area, we have installed scatterers that consist of a mix of 171.5 " in diameter solo PVC pipes, 11 pipe bundles of which has 100.5 " in diameter PVC pipes, and 30.25 " in diameter copper pipe. The target is $30.25^{\prime}$ in diameter copper pipes bundled by duct tapes. All the pipes are $10^{\prime}$ tall and fixed by anchors. The target is surrounded by the scatterers. When testing, we first measure the SAR phase history data for the clutter without the presence of the target. Then the second run of the data collection is conducted when the target is put back in.

\section{Imaging Results by Experimental Data}

Fig. 3 shows a SAR image of the target after the clutter subtraction. The ghost artifacts are visible around the target at location 14.5 meter in azimuth and 438.5 meter in range. The four corner reflectors are shown as four strong peaks. 


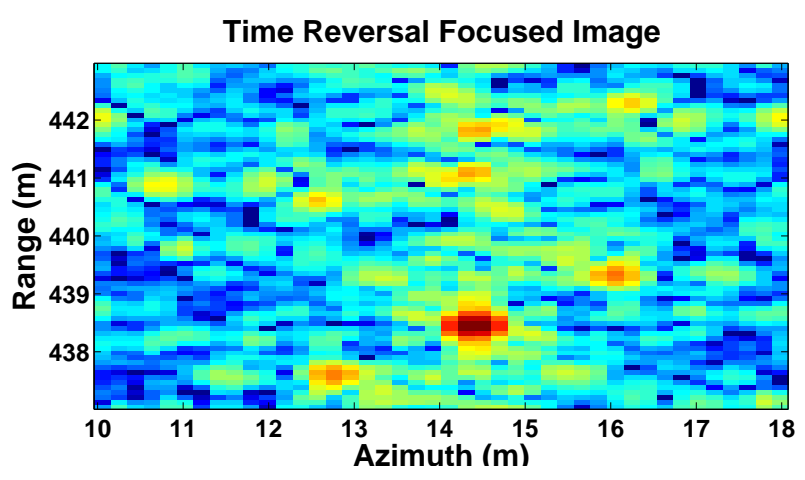

Figure 4. Time reversal focused SAR image

We process the image first by windowing the image in the image domain. We implement the windowing in order to remove the interference from the corner reflectors and to localize the multipath effect in the vicinity of the target of interest. The image chip will undergo TR-SAR processing to reconstruct the target image. Fig. 4 depicts the TR-SAR reconstructed image. To view the resolution, we plot the image projections in range and azimuth. In Fig. 5, the upper panel shows the range projection of the TR-focused image and the conventional image. The plots show that the TRfocused image has a deeper null and slightly narrower main beam, which shows a better resolution. The lower panel shows the range resolution; the difference is very small. This is because the system bandwidth remains the same for the two image formation methods.

\section{Conclusion and Continuing Work}

This paper describes and demonstrates time reversal algorithms for spotlight SAR under stretch processing in a rich scattering multipath environment. The rail-SAR data collected at the Rayethon test site show the benefits of TR-SAR that include improved resolution and reduced sidelobes and ghost artifacts. Further development will focus on the integration of TR-SAR into an automated target recognition (ATR) module in the SAR processing chain.

\section{Acknowledgement}

The authors would like to thank Dr. John Campbell, Ms. Laura Santos and other engineers at the Raytheon Missile Systems with experimental data collection.

\section{References}

[1] W. G. Carrara, R. S. Goodman, and R. M. Majewski, Spotlight Synthetic Aperture Radar: Signal Processing Algorithms. Boston, MA: Artech House, 1995.

[2] S. R. Deraaf, "SAR imaging via modern 2-D spectral estimation methods," IEEE Transactions on Image Processing, vol. 7, no. 5, pp. 729-761, May 1998.
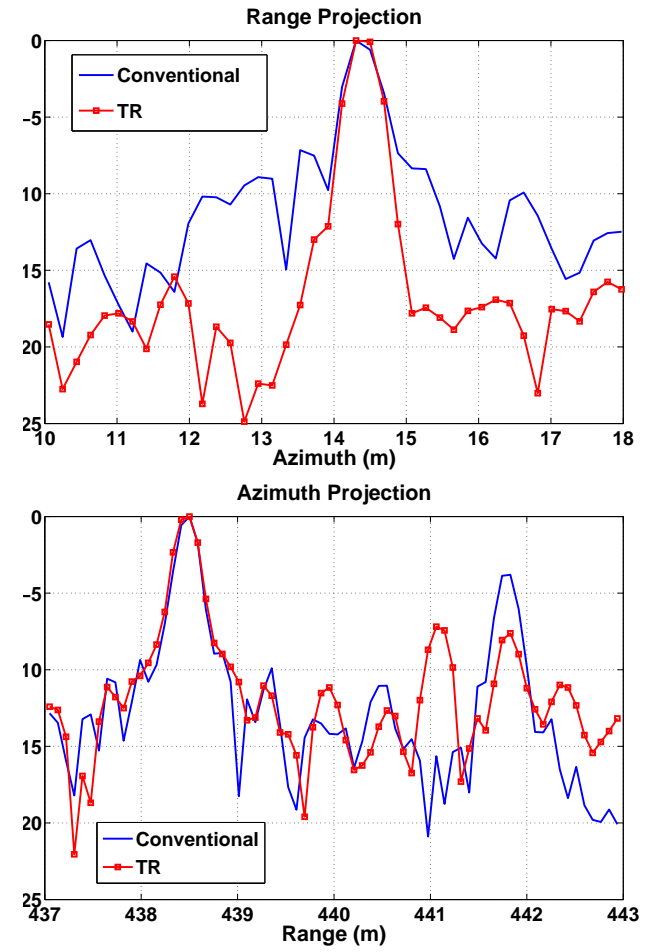

Figure 5. Image projections. Upper - Range projection; Lower - Azimuth projection.

[3] G. Benitz, "High definition vector imaging," Lincoln Laboratory Journal, vol. 10, no. 2, pp. 147-170, 1997.

[4] Y. Jin and J. M. F. Moura, "TR-SAR: time reversal target focusing in spotlight SAR," in ICASSP'07, IEEE International Conference on Signal Processing, vol. 2. Honolulu, HI: IEEE, April 2007, pp. 957-960.

[5] W. J. Caputi, "Stretch: A time transformation technique," IEEE Transactions on Aerospace and Electronic Systems, vol. AES-7, pp. 269-278, March 1971.

[6] P. Tait, Introduction to Radar Target Recognition. United Kingdom: The Institution of Electrical Engineers, 2005.

[7] C. V. Jakowatz, D. E. Wahl, P. H. Eichel, D. C. Ghiglia, and P. A. Thompson, Spotlight-Mode Synthetic Aperture Radar: A Signal Processing Approach. Boston, MA: Kluwer Academic Publishers, 1996.

[8] Y. Jin, J. M. F. Moura, Y. Jiang, J. Zhu, and D. Stancil, "Time reversal target focusing in spotlight SAR," in 15th Adaptive Sensor Array Processing (ASAP) Workshop. Lexington, MA: MIT Lincoln Lab, June 2007. 